\title{
Ultrasound method applied to characterize healthy femoral diaphysis of Wistar rats in vivo
}

\author{
A. Fontes-Pereira ${ }^{1}$, D.P. Matusin ${ }^{1}$, P. Rosa ${ }^{1}$, A. Schanaider ${ }^{2}$, M.A. von Krüger $^{1}$ and W.C.A. Pereira $^{1}$ \\ ${ }^{1}$ Programa de Engenharia Biomédica, Universidade Federal do Rio de Janeiro, Rio de Janeiro, RJ, Brasil \\ ${ }^{2}$ Departamento de Cirurgia, Escola de Medicina, Universidade Federal do Rio de Janeiro, Rio de Janeiro, RJ, Brasil
}

\begin{abstract}
A simple experimental protocol applying a quantitative ultrasound (QUS) pulse-echo technique was used to measure the acoustic parameters of healthy femoral diaphyses of Wistar rats in vivo. Five quantitative parameters [apparent integrated backscatter (AIB), frequency slope of apparent backscatter (FSAB), time slope of apparent backscatter (TSAB), integrated reflection coefficient (IRC), and frequency slope of integrated reflection (FSIR)] were calculated using the echoes from cortical and trabecular bone in the femurs of 14 Wistar rats. Signal acquisition was performed three times in each rat, with the ultrasound signal acquired along the femur's central region from three positions $1 \mathrm{~mm}$ apart from each other. The parameters estimated for the three positions were averaged to represent the femur diaphysis. The results showed that AIB, FSAB, TSAB, and IRC values were statistically similar, but the FSIR values from Experiments 1 and 3 were different. Furthermore, Pearson's correlation coefficient showed, in general, strong correlations among the parameters. The proposed protocol and calculated parameters demonstrated the potential to characterize the femur diaphysis of rats in vivo. The results are relevant because rats have a bone structure very similar to humans, and thus are an important step toward preclinical trials and subsequent application of QUS in humans.
\end{abstract}

Key words: Bone; Tissue characterization; Quantitative ultrasound; Clinical research protocols; Animal model

\section{Introduction}

Bone is formed by specialized connective tissue; its extracellular matrix is calcified but maintains a degree of elasticity $(1,2)$. Despite its hardness and resilience owing to an association between collagen and hydroxyapatite crystals $(2,3)$, bone injury is still a recurrent health condition. The process of fracture healing is complex $(3,4)$, involving cellular proliferation and differentiation, chemotaxis, and synthesis of extracellular matrix (3), with several stages of repair with a well-defined temporal and spatial sequence $(5,6)$. Severe complications frequently occur during the process of bone repair (7-9), such as delayed union, malunion, or nonunion (pseudarthrosis) (10), resulting in negative consequences for patients and increased costs to healthcare systems $(7,8)$, and justifying efforts to develop new diagnostic tools to track the bone healing process.

Protopappas et al. (11), using computational simulations and experiments (4), found that material properties and geometrical features change, influencing the propagation of ultrasound waves along the cortex of long bones. Dodd et al. (12) also pointed out that fracture gaps can promote loss of ultrasound energy. Hakulinen et al. (13) studied the measurement of bone density and mechanical properties by ultrasonic propagation parameters, reporting results that indicated a potential for diagnosing osteoporosis.

Application of quantitative ultrasound (QUS) to measure acoustic parameters of bone structure $(2,5,6,8,14$, 15) can enable the detection of changes and information about deviations from the normal condition caused by diseases $(14,16-18)$ such as osteoporosis $(13,19,20)$, osteomyelitis (10), and osteoarthritis. QUS can potentially minimize subjectivity in diagnoses made by conventional imaging methods. It has the advantage of low operating costs (13), ease in equipment handling $(16,21)$, and employing nonionizing radiation (22), unlike conventional X-ray and computed tomography (23). Although the literature reports innumerable uses of QUS for the characterization of soft biological tissue $(14,24)$, clinical

Correspondence: A. Fontes-Pereira, Programa de Engenharia Biomédica/COPPE, Centro de Tecnologia, Bloco H, Caixa Postal 68510, 21945-970 Rio de Janeiro, RJ, Brasil. Fax: +55-21-2562-8591. E-mail: aldo.fontes@gmail.com 
application is still modest (21) because of the lack of reproducibility of the method. Nevertheless, QUS is emerging as a promising modality for bone characterization (21). Methods to characterize bone have been developed using animal models, such as cattle $(19,25)$ and sheep $(11,26)$, and have been subsequently explored in humans $(15,18,20)$. Among animal bone models, few studies (27) have explored the use of QUS to quantify reflection and backscattering from the bones of Wistar rat models in vivo, which are the most similar to human bones.

The present study aimed to develop a QUS method using a pulse-echo technique to extract five parameters: apparent integrated backscatter (AIB), frequency slope of apparent backscatter (FSAB), time slope of apparent backscatter (TSAB), integrated reflection coefficient (IRC), and frequency slope of integrated reflection (FSIR) by processing ultrasonic backscattered and reflection echoes to characterize cortical and trabecular bone from healthy bone diaphyses of Wistar rats in vivo. Although there is no adequate standard characterization method for diaphyseal bone in the literature, there exist two previous conference papers published by our group on this subject $(27,28)$. To our knowledge, no other similar in vivo studies in rats have been published.

\section{Material and Methods}

The research was approved by the Ethics Committee for the Use of Laboratory Animals in Research of the Faculdade de Medicina, Universidade Federal do Rio de Janeiro. The animals were housed in accordance with the Guidelines for Care and Use of Animals in Research. The sample consisted of seven 3-month-old Wistar rats (Rattus norvegicus albinus) weighing $225 \pm 25$ g, previously anesthetized, and with their two hind legs shaved.

\section{Experimental setup}

The acquisition of ultrasound signals was performed by the same researcher three times in each animal at an interval of 10 days under the same environmental conditions and temperature $\left(22.5 \pm 1.1^{\circ} \mathrm{C}\right)$. The animals were killed following the last measurement.

The protocol for signal acquisition was as follows: a) Each animal was laid down in a lateral position with its hind limb relaxed and resting on a polished steel plate placed perpendicular to the ultrasonic beam axis. b) The transducer was held vertically by a stereotactic holder of a $2-\mu \mathrm{m}$ resolution, with the beam focused on the femur diaphysis (region of interest). c) The coupling of transducer to the limb covered with a water-soluble gel was made with a waveguide, a glass tube filled with degassed water sealed by $10.5-\mu \mathrm{m}$ thick polyvinyl chloride. d) Adjustment of the transducer position was guided by palpation (the greater trochanter and lateral condyle of the femur were found to define the lateral middle third of the femur) and by maximizing the echo signals (displayed on an oscilloscope screen). The central point position corresponded to the maximum echo and two other points, displaced $1 \mathrm{~mm}$ to each side, were also chosen. Signals from the three points were recorded.

A reference signal from a polished steel plate $(1 \mathrm{~cm}$ thick) was acquired by pointing the waveguide to the plate's front surface, which was covered with gel, but without the animal sample.

Soft tissue thickness was measured with a B-mode scanning VEVO ${ }^{\circledR} 770$ (VisualSonics, Inc., Canada) transducer at $30 \mathrm{MHz}$. The time position of the echo from the muscle/bone interface was identified in the radiofrequency (RF) signal, according to the transducer-bone distance calculated from the B-mode images. The experiment was carried out three times, with an interval of 10 days, and the experimental setup was rebuilt each time before measurements took place.

\section{Measurements of ultrasound parameters}

The transducer (model V326, Olympus ${ }^{\mathbb{R}}$ NDT, Inc., USA) with a nominal frequency of $5 \mathrm{MHz}, 9.5 \mathrm{~mm}$ in diameter, and $69.3 \mathrm{~mm}$ in focal length, was driven by a pulse generator (model SR9000, Matec ${ }^{\circledR}$, Inc., USA). The echoes were displayed and measured on an oscilloscope (model TDS 2024B, Tektronix ${ }^{\circledR}$, Inc., USA).

The RF echo segment (Figure 1) used for characterization of the bone diaphysis consisted of the following: i) the echo from the bone surface and ii) the backscattering signal of the inner bone. The window containing this segment was determined by identifying the echo from the bone surface, which corresponded to the reference echo from the steel plate, and selecting a first rectangular window encompassing its limits using a $10 \%$ peak amplitude threshold (Figure 2). The segment of $4-\mu \mathrm{s}$ duration containing the backscattered signal from the

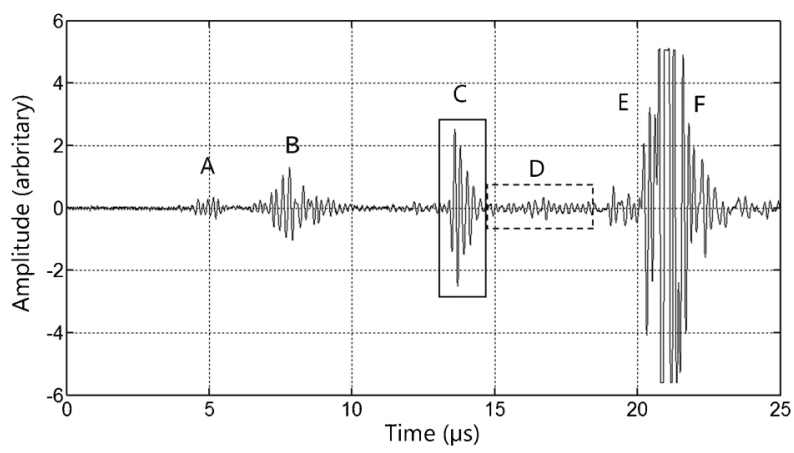

Figure 1. Radiofrequency signal displaying the echo interfaces. $A$, Degassed water/PVC membrane interface; $B$, skin/fat/muscle interface; $C$, window from surface bone (reflection, continuous line box); $D$, window from inside bone (backscatter, dotted line box); $E$, surface bone/skin/fat/muscle interface (limb/gel); $F$, skin/ reflective plate interface. 


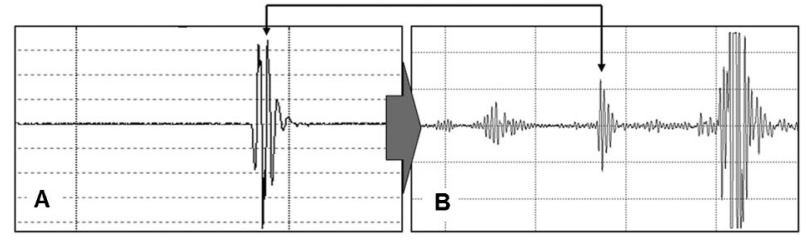

Figure 2. A, Definition of the rectangular window over the reflected echo from the reference steel plate (extreme limits on $10 \%$ of peak amplitude). $B$, Reference rectangular window placed over the echo from interface muscle/bone. The remaining echoes on the right with a $4-\mu$ s duration are from backscattering inside the bone.

inside of the bone (which has no interference from the muscle/bone interface echo) began from the end of this window. This time duration was chosen to ensure that the backscattering signal came from within the bone, which, in adult Wistar rats, has an estimated average diameter of $3.12 \pm 0.1 \mathrm{~mm}$.

Five parameters were used to characterize the echoes from specimen and reference signals: AIB, FSAB, TSAB, IRC, and FSIR, and an algorithm was developed in Matlab ${ }^{\mathbb{R}}$ code (MathWorks, Inc., USA) to calculate these parameters. The apparent backscatter transfer function (ABTF; Equation 1) was obtained from the literature (20) where $P_{\text {reference }}$ and $P_{\text {specimen }}$ are the power spectra of reference and specimen signals (rectangular window from bone surface), respectively.

$A B T F=10 \log _{10} P_{\text {specimen }}(f)-10 \log _{10} P_{\text {reference }}(f)$

AIB, determined by integrating the curve of ABTF (Equation 2), expresses the average value of apparent backscattering in the frequency range of interest, while $F S A B$, the slope of the linear regression line obtained from the curve ABTF vs frequency, is the fraction of apparent backscattering corresponding to each frequency. Furthermore, TSAB, the slope of the linear regression of values of AIB as a function of time, is the variation of apparent backscattering as the wave propagates through the tissue.

$A I B=\frac{\int_{f_{\text {low }}}^{f_{\text {high }}}[A B T F] . d f}{f_{\text {high }}-f_{\text {low }}}$

Reflection transfer function (RTF; Equation 3) was calculated from the rectangular window with the reflection signal, which has a definition similar to ABTF, while IRC is the average value of reflection within a frequency bandwidth, which is obtained by integrating RTF. FSIR is the slope of the linear regression of IRC and is the apparent fraction of the reflection corresponding to each frequency, and calculated similar to FSAB (20).

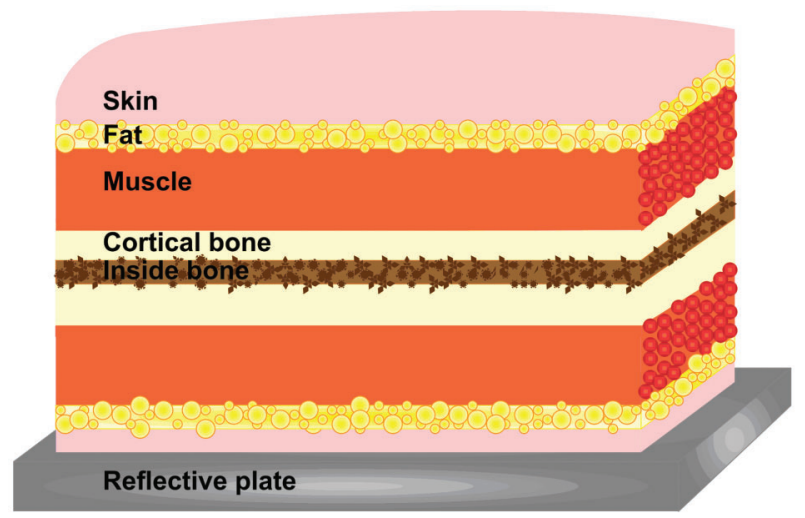

Figure 3. Layers of biological tissues (skin, fat, muscle, cortical bone, inside bone) and reflective plate.

$$
R T F=10 \log _{10} P_{\text {specimen }}(f)-10 \log _{10} P_{\text {reference }}(f)
$$

It must be noted that, before the estimation of parameters, all RF echoes must have their amplitudes corrected for loss in amplitude because of attenuation in the propagation path. By assuming that the medium is composed of four layers (skin, fat, muscle, and bone; Figure 3), the compensation factor $A_{f}$ can be written as follows (Equation 4): where $s, f$, and $m$ indices correspond to skin, fat, and muscle layers, respectively, while $\alpha s$, $\alpha$, and $\alpha m$ denote their respective attenuation coefficients, and $x s, x f$, and $x m$ indicate their respective thicknesses. Similarly, $T_{s / f}$ and $T_{f / m}$ are the transmission coefficients for the skin/fat and fat/muscle interfaces, respectively. The compensation factor takes into account the propagation to and from the bone surface.

$A_{f}=e^{2 \alpha s . x s} T_{s / f}^{2} e^{2 \alpha f \cdot x f} T_{f / m}^{2} e^{2 a m . x m}$

Five parameters were calculated from the three signals for each femur, and the average of these values was considered as representative of each hind limb. Thus, each femur was characterized by five parameters.

\section{Quantitative computed tomography (QCT) acquisition}

QCT (75 kV, $145 \mathrm{mAs}$ ) was performed with Triumph II PET/SPECT/micro-CT equipment (Gamma Medica, Inc., Canada). Eight femurs were put in the scanning plate in a lateral position (the same one used for ultrasound acquisition). The tomographic images were processed with the Osirix software for bone density analysis (in Hounsfield units) of the femur diaphysis. These data were used as the gold standard for bone density.

\section{Statistical analysis}

The Kolmogorov-Smirnov and equal variance tests were used to test normality; when these tests failed, the 
Table 1. Average values and standard deviation of each parameter in three experiments.

\begin{tabular}{lccccc}
\hline Experiments & AIB $(\mathrm{dB})$ & FSAB $(\mathrm{dB} / \mathrm{MHz})$ & TSAB $(\mathrm{dB} / \mu \mathrm{s})$ & $\mathrm{IRC}(\mathrm{dB})$ & $\mathrm{FSIR}(\mathrm{dB} / \mathrm{MHz})$ \\
\hline 1 & $-44.46 \pm 2.23$ & $-1.43 \pm 0.28$ & $-1.31 \pm 0.23$ & $-28.07 \pm 1.17$ & $-1.62 \pm 0.08$ \\
2 & $-43.96 \pm 1.09$ & $-1.39 \pm 0.27$ & $-1.30 \pm 0.16$ & $-28.13 \pm 0.73$ & $-1.63 \pm 0.05$ \\
3 & $-43.76 \pm 1.93$ & $-1.31 \pm 0.26$ & $-1.22 \pm 0.18$ & $-28.46 \pm 0.85$ & $-1.66 \pm 0.06$ \\
\hline
\end{tabular}

AIB: apparent integrated backscatter; FSAB: frequency slope of apparent backscatter; TSAB: time slope of apparent backscatter; IRC: integrated reflection coefficient; FSIR: frequency slope of integrated reflection.

nonparametric Friedman test was used. To test the null hypothesis that all parameters belonged to the same population, and to assess the reproducibility of the parameters and method used, a statistical analysis was performed by one-way repeated measures analysis of variance (ANOVA) at a $5 \%$ level of significance. The tests were carried out using SigmaStat 3.5 (Systat Software, Inc., USA). Pearson's correlation coefficient was used to quantify the correlation between the reflection parameters (IRC and FSIR) and surface bone density (as measured by QCT). Pearson's correlation was also used to evaluate the level of association between the ultrasonic parameters

\section{Results}

The average values and standard deviations of parameters AIB, FSAB, TSAB, IRC, and FSIR for each experiment are shown in Table 1.

Pearson's correlation coefficient showed a positive correlation between surface bone density and IRC (Figure 4A) and FSIR (Figure 4B) for the three experiments.

To determine the interaction between the parameters from backscatter (AIB, FSAB, and TSAB; Figure 5) and reflection (IRC and FSIR) of bones, Pearson's correlation was applied to parameters of Experiment 1 (Table 2), Experiment 2 (Table 3), and Experiment 3 (Table 4). The oneway repeated measures ANOVA was used independently to test the AIB, FSAB, TSAB, and FSIR data from the three experiments, which showed that $\operatorname{AIB}[F(2,41)=1.02$; $\mathrm{P}=0.37]$, FSAB $[F(2,41)=1.92 ; \mathrm{P}=0.17]$, and $\mathrm{TSAB}$ $[F(2,41)=1.61 ; P=0.22]$ belong to the same population With regard to the FSIR parameter, the differences in mean values among the experiments were greater than those expected, thus there was a statistically significant difference $[F(2,41)=4.17 ; P=0.03]$. To isolate the experiment that differed from the others, a pairwise multiple comparison procedure (Holm-Sidak method) at a $5 \%$ level of significance was employed, which showed that the FSIR parameter of Experiment 1 was different from that of Experiment $3(\mathrm{P}=0.01)$.

The IRC parameter did not follow a normal distribution, and thus we used the Friedman test at a $5 \%$ level of significance, which showed that this parameter belonged to the same population $[F(2)=4 ; P=0.14]$. Pearson's correlation coefficient showed, in general, strong correlations among the parameters.

\section{Discussion}

The literature on new protocols and tools for the characterization of bone by QUS is extensive (19$21,26,28)$, but, to date, there is no standardization of methods. In previous studies, several analyses have been conducted, including mathematical simulations (11), in vitro studies $(12,13,25)$, and even animal models
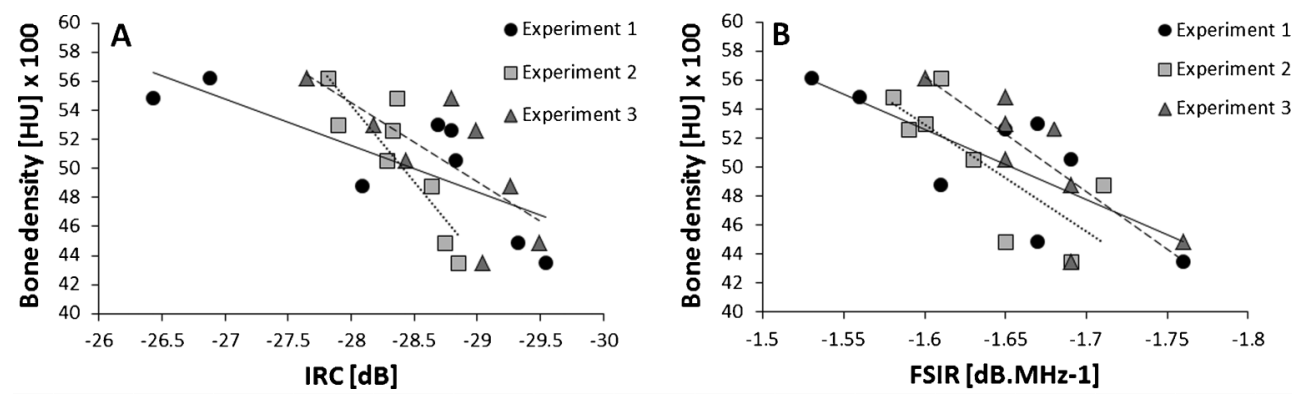

Figure 4. Pearson's correlation between surface bone density, the integrated reflection coefficient (IRC; $A$ ) and frequency slope of integrated reflection (FSIR; $B$ ) for three experiments. The experiment showed positive correlation between surface bone density and the parameter IRC $(r=0.79, P=0.019 ; r=0.87, P=0.005 ; r=0.724, P=0.042)$, in Experiments 1,2 , and 3 , respectively, and the parameter FSIR $(r=0.78 ; P=0.023 ; r=0.77 ; P=0.026 ; R=0.81 ; P=0.016)$, in Experiments 1,2 , and 3 , respectively. 

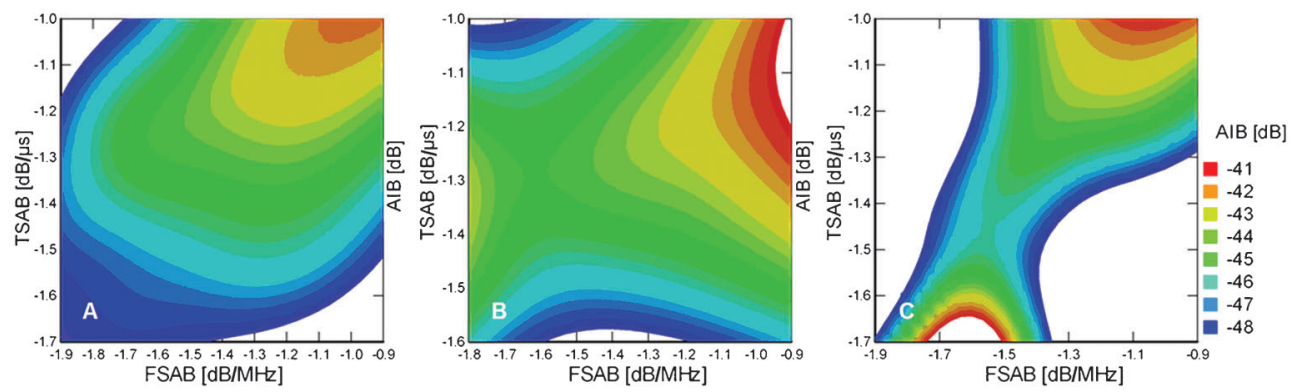

Figure 5. Correlation between the backscatter parameters (AIB, FSAB, and TSAB) in Experiments 1-3 of the bone. AIB: apparent integrated backscatter; FSAB: frequency slope of apparent backscatter; TSAB: time slope of apparent backscatter.

$(26,28)$, with different degrees of success. Even the promising studies are noted to have difficulties in extrapolating their results for use in preclinical trials. The following are some of the reasons for this limitation. The bone specimens examined had important differences, when compared with human bone $(17,19,25)$. The simulation models were very simple, or the method cannot be applied in vivo; and most of all, there is an important lack of reproducibility. When dealing with human in vivo experiments, studies are carried out mostly in a transmission mode applied to extremities (calcaneus, phalange, and forearm) (21), and the same difficulty is encountered with regard to definition of protocols as well as reproducibility.

QUS can help in minimizing the subjectivity of bone imaging and characterization and is more accurate for diagnosis and monitoring either the evolution of treatment or the course of metabolic diseases. In the present study, an in vivo experiment was proposed along with standardization and consolidation of protocols as a step prior to clinical use in humans. The first proposal was the use of live adult Wistar rats that, in addition to their easy handling and resistance in research $(9,29)$, have bone tissue characteristics more similar to human bones $(29,30)$ than other animals, with the exception of primates. Furthermore, as rats play an important role in the evaluation of metabolic bone diseases (29) and pathophysiological conditions $(29,30)$, the results are more likely to be similar to those expected in humans. Because rat femurs are small, it was necessary to use a $5-\mathrm{MHz}$ transducer to provide satisfactory resolution (20). Additionally, the precise positioning of the ultrasonic beam at the three acquisition sites on the bone, chosen close enough to ensure minimal anatomical variations, was ensured by a high-precision stereotactic holder. The acquisition of in vivo signals is highly influenced by soft tissue attenuation and reflection; therefore, a compensation factor $\left(A_{f}\right)$ to reduce measurement errors (31) was adopted.

QCT provides accurate bone density measurements of the cortical bone that is used as a gold standard. In the three experiments, a positive correlation between the IRC and FSIR parameters and surface bone density was identified. According to the value correlation coefficient, we consider our method as adequate to characterize cortical bone density.

The five parameters estimated were shown to be appropriate for characterizing the rat femurs in vivo. It is interesting to note that the first three parameters were described by Hoffmeister et al. (20) for in vitro characterization of human cancellous bones. On the other hand, IRC was used for in vitro characterization of trabecular bovine bone $(13,32)$ and in vitro trabecular human bone $(31,33)$, and FSIR was developed by our group for characterization of rat bone. The AIB parameter can be used to characterize soft tissues (24). Hoffmeister et al. (20) used AIB $(-40.9 \pm 2.0 \mathrm{~dB})$, FSAB $(-1.7 \pm 0.5 \mathrm{~dB} / \mathrm{MHz})$, and TSAB

Table 2. Pearson's correlation coefficient $(r)$ of parameters and $\mathrm{P}$ for Experiment 1.

\begin{tabular}{|c|c|c|c|c|c|}
\hline & AIB $(d B)$ & $\mathrm{FSAB}(\mathrm{dB} / \mathrm{MHz})$ & $\mathrm{TSAB}(\mathrm{dB} / \mu \mathrm{s})$ & $\operatorname{IRC}(\mathrm{dB})$ & $\mathrm{FSIR}(\mathrm{dB} / \mathrm{MHz})$ \\
\hline $\mathrm{AIB}(\mathrm{dB})$ & - & $r=0.92^{\mathrm{c}}$ & $r=0.98^{d}$ & $r=-0.87^{\mathrm{b}}$ & $r=-0.83^{\mathrm{a}}$ \\
\hline $\mathrm{FSAB}(\mathrm{dB} / \mathrm{MHz})$ & $r=0.92^{\mathrm{C}}$ & - & $r=0.89^{b}$ & $r=-0.93^{\mathrm{c}}$ & $r=-0.86^{\mathrm{b}}$ \\
\hline TSAB (dB/ $/ \mu \mathrm{s})$ & $r=0.98^{\mathrm{d}}$ & $r=0.89^{b}$ & - & $r=-0.86^{\mathrm{b}}$ & $r=-0.79^{a}$ \\
\hline $\operatorname{IRC}(\mathrm{dB})$ & $r=-0.87^{\mathrm{b}}$ & $r=-0.93^{\mathrm{c}}$ & $r=-0.86^{\mathrm{b}}$ & - & $r=0.85^{a}$ \\
\hline
\end{tabular}

${ }^{\mathrm{a}} \mathrm{P}<0.001$; ${ }^{\mathrm{b}} \mathrm{P}<0.0001$; ${ }^{\mathrm{c}} \mathrm{P}<0.00001 ;{ }^{\mathrm{d}} \mathrm{P}<0.00000001$. AIB: apparent integrated backscatter; FSAB: frequency slope of apparent backscatter; TSAB: time slope of apparent backscatter; IRC: integrated reflection coefficient; FSIR: frequency slope of integrated reflection. 
Table 3. Pearson's correlation coefficient $(r)$ of parameters and $\mathrm{P}$ for Experiment 2.

\begin{tabular}{|c|c|c|c|c|c|}
\hline & AIB $(d B)$ & FSAB $(\mathrm{dB} / \mathrm{MHz})$ & $\mathrm{TSAB}(\mathrm{dB} / \mu \mathrm{s})$ & $\operatorname{IRC}(\mathrm{dB})$ & $\mathrm{FSIR}(\mathrm{dB} / \mathrm{MHz})$ \\
\hline AIB (dB) & - & $r=0.69^{\mathrm{e}}$ & $r=0.86^{g}$ & $r=-0.56^{d}$ & $r=-0.33^{a}$ \\
\hline $\mathrm{FSAB}(\mathrm{dB} / \mathrm{MHz})$ & $r=0.69^{\mathrm{e}}$ & - & $r=0.77^{\mathrm{e}}$ & $r=-0.72^{\mathrm{e}}$ & $r=-0.66^{\mathrm{e}}$ \\
\hline $\mathrm{TSAB}(\mathrm{dB} / \mu \mathrm{s})$ & $r=0.86^{\mathrm{g}}$ & $r=0.77^{\mathrm{e}}$ & - & $r=-0.50^{\mathrm{c}}$ & $r=-0.37^{\mathrm{b}}$ \\
\hline IRC (dB) & $r=-0.56^{\mathrm{d}}$ & $r=0.72^{\mathrm{e}}$ & $r=-0.50^{\mathrm{c}}$ & - & $r=0.81^{\mathrm{f}}$ \\
\hline
\end{tabular}

${ }^{\mathrm{a}} \mathrm{P}=0.24 ;{ }^{\mathrm{b}} \mathrm{P}=0.19 ;{ }^{\mathrm{c}} \mathrm{P}=0.07 ;{ }^{\mathrm{d}} \mathrm{P}<0.05 ;{ }^{\mathrm{e}} \mathrm{P}<0.01 ;{ }^{\mathrm{f}} \mathrm{P}<0.001 ;{ }^{\mathrm{g}} \mathrm{P}<0.0001$. AlB: apparent integrated backscatter; FSAB: frequency slope of apparent backscatter; TSAB: time slope of apparent backscatter; IRC: integrated reflection coefficient; FSIR: frequency slope of integrated reflection.

$(-4.2 \pm 0.6 \mathrm{~dB} / \mu \mathrm{s})$ to characterize in vitro human trabecular bone, and reported promising results. In our research, the lower values of our AIB parameter, when compared with the literature (20), can perhaps be explained by the effects of cortical bone, which increases the loss of ultrasonic energy by reflection; therefore, less energy was transmitted to the inside of the bone, and, hence, the energy backscattered from this region was decreased. FSAB, on the other hand, had higher (less negative) values, when compared with the literature (20), indicating that, although the overall energy backscattered was smaller, more energy from higher frequencies was backscattered. Similar behavior was observed with respect to TSAB, where more energy was backscattered from deeper structures, thus giving less negative TSAB values than those in the literature (20). The low standard deviations for parameters AIB, FSAB, and TSAB suggest that our method gave statistically similar results at different points along the femur; this is clinically significant, considering the surface anatomical irregularities of the bone.

IRC $(13,31,32)$ represents the amount of reflection from a tissue; thus, a denser bone tends to have higher values of IRC. Hakulinen et al. (13), using bovine trabecular bones in vitro from different regions of the femur, found the following values for the parameter IRC: $-17.9 \pm 3.9 \mathrm{~dB}$ (medial condyle), $-20.3 \pm 3.8 \mathrm{~dB}$ (lateral condyle), and $-27.2 \pm 2.0 \mathrm{~dB}$ (greater trochanter). The lower values of IRC observed in our research may be because the study had a layer of soft tissue that strongly influenced the ultrasonic parameters (33); however, when we conducted in vitro experiments (34), we found results similar to those in the literature.

We introduced the parameter FSIR as a new way to characterize reflection as a function of frequency (28). FSIR showed strong positive correlation to IRC in the three experiments, which suggests that this parameter is related to the density of cortical bone and is promising among the other parameters for monitoring the process of bone healing. IRC had a negative correlation to the backscatter parameters (except in two cases), indicating that it can be an additional parameter for assessing bone quality. Furthermore, IRC was negatively correlated to parameter AIB. This fact indicates that the method proposed in this study succeeded in characterizing the surface and inside of the bone.

The experiments were performed at three different periods, with intervals of 10 days, to study the repeatability of the method. The statistical tests showed no difference between parameters AIB, FSAB, TSAB, and IRC obtained from the three experiments, but the FSIR values obtained in Experiment 1 and Experiment 3 were significantly different. This difference might have been caused by errors of signal acquisition in a different region of interest. Statistical analysis of the correlation between the parameters indicated that there was lack of correlation in only three cases in Experiment 2; however, overall, the experiments suggested that all the parameters have the potential to characterize bone.

The proposed protocol and calculated parameters

Table 4. Pearson's correlation coefficient $(r)$ of parameters and $\mathrm{P}$ for Experiment 3.

\begin{tabular}{|c|c|c|c|c|c|}
\hline & AIB $(d B)$ & $\mathrm{FSAB}(\mathrm{dB} / \mathrm{MHz})$ & $\mathrm{TSAB}(\mathrm{dB} / \mu \mathrm{s})$ & $\operatorname{IRC}(\mathrm{dB})$ & $\mathrm{FSIR}(\mathrm{dB} / \mathrm{MHz})$ \\
\hline $\mathrm{AIB}(\mathrm{dB})$ & - & $r=0.96^{\mathrm{e}}$ & $r=0.97^{\mathrm{e}}$ & $r=-0.95^{\mathrm{d}}$ & $r=-0.86^{\mathrm{b}}$ \\
\hline $\mathrm{FSAB}(\mathrm{dB} / \mathrm{MHz})$ & $r=0.96^{\mathrm{e}}$ & - & $r=0.96^{\mathrm{e}}$ & $r=-0.90^{\mathrm{b}}$ & $r=-0.85^{\mathrm{a}}$ \\
\hline TSAB (dB/ $/ \mu \mathrm{s})$ & $r=0.97^{\mathrm{e}}$ & $r=0.96^{\mathrm{e}}$ & - & $r=-0.93^{\mathrm{c}}$ & $r=-0.85^{\mathrm{a}}$ \\
\hline $\operatorname{IRC}(\mathrm{dB})$ & $r=-0.95^{\mathrm{d}}$ & $r=-0.90^{\mathrm{b}}$ & $r=-0.93^{\mathrm{c}}$ & - & $r=0.90^{\mathrm{b}}$ \\
\hline
\end{tabular}

${ }^{\mathrm{a}} \mathrm{P}<0.001 ;{ }^{\mathrm{b}} \mathrm{P}<0.0001 ;{ }^{\mathrm{c}} \mathrm{P}<0.00001 ;{ }^{\mathrm{d}} \mathrm{P}<0.000001$; ${ }^{\mathrm{e}} \mathrm{P}<0.0000001$. AIB: apparent integrated backscatter; FSAB: frequency slope of apparent backscatter; TSAB: time slope of apparent backscatter; IRC: integrated reflection coefficient; FSIR: frequency slope of integrated reflection. 
demonstrate the potential to characterize femur diaphysis of rats in vivo by the pulse-echo ultrasonic method associated with wave reflection and backscattering. The values of the five parameters in rats, as well as the simple protocol for signal acquisition, provide supplementary predictive data of living human bones. Furthermore, this research contributes to the use of rats in vivo in future studies of bone characterization to provide data for more

\section{References}

1. Rho JY, Kuhn-Spearing L, Zioupos P. Mechanical properties and the hierarchical structure of bone. Med Eng Phys 1998; 20: 92-102, doi: 10.1016/S1350-4533(98)00007-1.

2. Marks SC, Odgren PR. Structure and development of the skeleton. In: Bilezikian JP, Raisz LG, Rodan GA (Editors), Principles of bone biology. 1st edn. San Diego: Academic Press; 1996. p 3-14.

3. Feng X, McDonald JM. Disorders of bone remodeling. Annu Rev Pathol 2011; 6: 121-145, doi: 10.1146/annurev-pathol011110-130203.

4. Protopappas VC, Baga DA, Fotiadis DI, Likas AC, Papachristos AA, Malizos KN. An ultrasound wearable system for the monitoring and acceleration of fracture healing in long bones. IEEE Trans Biomed Eng 2005; 52: 1597-1608, doi: 10.1109/TBME.2005.851507.

5. Dimitriou R, Tsiridis E, Giannoudis PV. Current concepts of molecular aspects of bone healing. Injury 2005; 36: 13921404, doi: 10.1016/j.injury.2005.07.019.

6. Phillips AM. Overview of the fracture healing cascade. Injury 2005; 36 (Suppl 3): S5-S7, doi: 10.1016/j.injury.2005.07.027.

7. Kanakaris NK, Giannoudis PV. The health economics of the treatment of long-bone non-unions. Injury 2007; 38 (Suppl 2): S77-S84, doi: 10.1016/S0020-1383(07)80012-X.

8. Rose FR, Oreffo RO. Bone tissue engineering: hope vs hype. Biochem Biophys Res Commun 2002; 292: 1-7, doi: 10.1006/bbrc.2002.6519.

9. Ferreira ML, Silva PC, Alvarez Silva LH, Bonfim DC, Conilho Macedo Muller LC, Esposito CC, et al. Heterologous mesenchymal stem cells successfully treat femoral pseudarthrosis in rats. J Trans/ Med 2012; 10: 51, doi: 10.1186/1479-5876-10-51.

10. Jackson LC, Pacchiana PD. Common complications of fracture repair. Clin Tech Small Anim Pract 2004; 19: 168179, doi: 10.1053/j.ctsap.2004.09.008.

11. Protopappas VC, Fotiadis DI, Malizos KN. Guided ultrasound wave propagation in intact and healing long bones. Ultrasound Med Biol 2006; 32: 693-708, doi: 10.1016/ j.ultrasmedbio.2006.02.001.

12. Dodd SP, Cunningham JL, Miles AW, Gheduzzi S, Humphrey VF. An in vitro study of ultrasound signal loss across simple fractures in cortical bone mimics and bovine cortical bone samples. Bone 2007; 40: 656-661, doi: 10.1016/j.bone.2006. 10.004.

13. Hakulinen MA, Toyras J, Saarakkala S, Hirvonen J, Kroger $\mathrm{H}$, Jurvelin JS. Ability of ultrasound backscattering to predict mechanical properties of bovine trabecular bone. Ultrasound Med Biol 2004; 30: 919-927, doi: 10.1016/j.ultrasmedbio. 2004.04.006.

14. Meziri M, Pereira WC, Abdelwahab A, Degott C, Laugier P. consistent computer simulations.

\section{Acknowledgments}

The authors are grateful to Dr. Célia Resende for performing the ultrasonographies and to Dr. Sergio Souza for computed tomography acquisition. Research supported by CNPq, CAPES, and FAPERJ.

In vitro chronic hepatic disease characterization with a multiparametric ultrasonic approach. Ultrasonics 2005; 43 : 305-313, doi: 10.1016/j.ultras.2004.09.002.

15. Zehnder $\mathrm{Y}$, Luthi M, Michel D, Knecht H, Perrelet R, Neto I, et al. Long-term changes in bone metabolism, bone mineral density, quantitative ultrasound parameters, and fracture incidence after spinal cord injury: a cross-sectional observational study in 100 paraplegic men. Osteoporos Int 2004; 15 180-189, doi: 10.1007/s00198-003-1529-6.

16. Joseph AE, Saverymuttu SH, al-Sam S, Cook MG, Maxwell JD. Comparison of liver histology with ultrasonography in assessing diffuse parenchymal liver disease. Clin Radiol 1991; 43: 26-31, doi: 10.1016/S0009-9260(05)80350-2.

17. Fontes-Pereira AJ, Teixeira RC, Oliveira AJ, Pontes RW, Barros RS, Negrão JN. The effect of low-intensity therapeutic ultrasound in induced fracture of rat tibiae. Acta Ortop Bras 2013; 21: 18-22, doi: 10.1590/S1413-78522013000100003.

18. Baroncelli GI, Federico G, Bertelloni S, Sodini F, De Terlizzi F, Cadossi R, et al. Assessment of bone quality by quantitative ultrasound of proximal phalanges of the hand and fracture rate in children and adolescents with bone and mineral disorders. Pediatr Res 2003; 54: 125-136, doi: 10.1203/01.PDR.0000069845.27657.EB.

19. Njeh CF, Boivin CM, Langton CM. The role of ultrasound in the assessment of osteoporosis: a review. Osteoporos Int 1997; 7: 7-22, doi: 10.1007/BF01623454.

20. Hoffmeister BK, Johnson DP, Janeski JA, Keedy DA, Steinert BW, Viano AM, et al. Ultrasonic characterization of human cancellous bone in vitro using three different apparent backscatter parameters in the frequency range 0.6$15.0 \mathrm{MHz}$. IEEE Trans Ultrason Ferroelectr Freq Control 2008; 55: 1442-1452, doi: 10.1109/TUFFC.2008.819.

21. Laugier $P$. Instrumentation for in vivo ultrasonic characterization of bone strength. IEEE Trans Ultrason Ferroelectr Freq Control 2008; 55: 1179-1196, doi: 10.1109/TUFFC.2008.782.

22. Dalecki D. Mechanical bioeffects of ultrasound. Annu Rev Biomed Eng 2004; 6: 229-248, doi: 10.1146/annurev.bioeng. 6.040803.140126.

23. Freeman TA, Patel P, Parvizi J, Antoci V Jr, Shapiro IM Micro-CT analysis with multiple thresholds allows detection of bone formation and resorption during ultrasound-treated fracture healing. J Orthop Res 2009; 27: 673-679, doi: 10.1002/jor.20771.

24. Bridal SL, Fornes P, Bruneval P, Berger G. Parametric (integrated backscatter and attenuation) images constructed using backscattered radio frequency signals (25$56 \mathrm{MHz}$ ) from human aortae in vitro. Ultrasound Med Biol 1997; 23: 215-229, doi: 10.1016/S0301-5629(96)00201-3.

25. Lee KI. Relationships of bone density with quantitative 
ultrasound parameter in bovine cancellous bone. J Korean Phys Soc 2009; 54: 71-74, doi: 10.3938/jkps.54.71.

26. Barbieri G, Mazzer N, Ribeiro EA, Nogueira-Barbosa MH, Barbieri $\mathrm{CH}$. A comparative analysis between ultrasonometry and computer-aided tomography to evaluate bone healing. J Orthop Res 2012; 30: 1076-1082, doi: 10.1002/ jor.22039.

27. Pereira AJF, Matusin DP, Rosa PTCR, Machado CB, Ferreira ML, Schanaider A, et al. In vivo characterization of long-bone in animal model by two ultrasonic scattering parameters: $A I B$ and FSAB. 358, Rio de Janeiro: Pan American Health Care Exchanges; 2011.

28. Pereira AJF, Costa TCFV, Schanaider A, Von Kruger MA, Pereira WCA. In vivo characterization of long-bone in animal model by ultrasonic reflection parameters: IRC and FSIR. Miami: Pan American Health Care Exchanges; 2012.

29. Blouin S, Basle MF, Chappard D. Rat models of bone metastases. Clin Exp Metastasis 2005; 22: 605-614, doi: 10.1007/s10585-006-9002-5.

30. Lelovas PP, Xanthos TT, Thoma SE, Lyritis GP, Dontas IA.
The laboratory rat as an animal model for osteoporosis research. Comp Med 2008; 58: 424-430.

31. Riekkinen $\mathrm{O}$, Hakulinen MA, Timonen M, Toyras J, Jurvelin JS. Influence of overlying soft tissues on trabecular bone acoustic measurement at various ultrasound frequencies. Ultrasound Med Biol 2006; 32: 1073-1083, doi: 10.1016/ j.ultrasmedbio.2006.03.012.

32. Hakulinen MA, Day JS, Toyras J, Weinans H, Jurvelin JS. Ultrasonic characterization of human trabecular bone microstructure. Phys Med Biol 2006; 51: 1633-1648, doi: 10.1088/0031-9155/51/6/019.

33. Riekkinen O, Hakulinen MA, Lammi MJ, Jurvelin JS, Kallioniemi A, Toyras J. Acoustic properties of trabecular bone - relationships to tissue composition. Ultrasound Med Biol 2007; 33: 1438-1444, doi: 10.1016/j.ultrasmedbio. 2007.04.004

34. Pereira AJF, Matusin DP, Machado CB, Ferreira ML, Schanaider A, Pereira WCA. Comparison of in vivo and in vitro long bone ultrasound characterization in animal model. Amsterdam: Physiotherapy; 2011. 